\title{
Erratum to: The current status and the future of JAK2 inhibitors for the treatment of myeloproliferative diseases
}

Yasumichi Hitoshi • Nan Lin • Donald G. Payan •

Vadim Markovtsov

Published online: 18 March 2010

(C) The Japanese Society of Hematology 2010

Erratum to: Int J Hematol (2010) 91:189-200

DOI 10.1007/s12185-010-0531-y

Errors appeared in Fig. 1. In Fig. 1a, in the two right panels the red arrowheads should be double-headed arrows. The relevant part of the figure legend should read: Red double-headed arrows indicate the distance between the G-loops of JAK2 and SYK. In Fig. 1b the leader lines identifying amino acids in the left and center panels of the figure were inadvertently omitted. The correct figure appears here.

Also, in the second column on p. 190, lines 4 and 5 should read: "...the ATP-binding site of JAKs, and the angle of the....
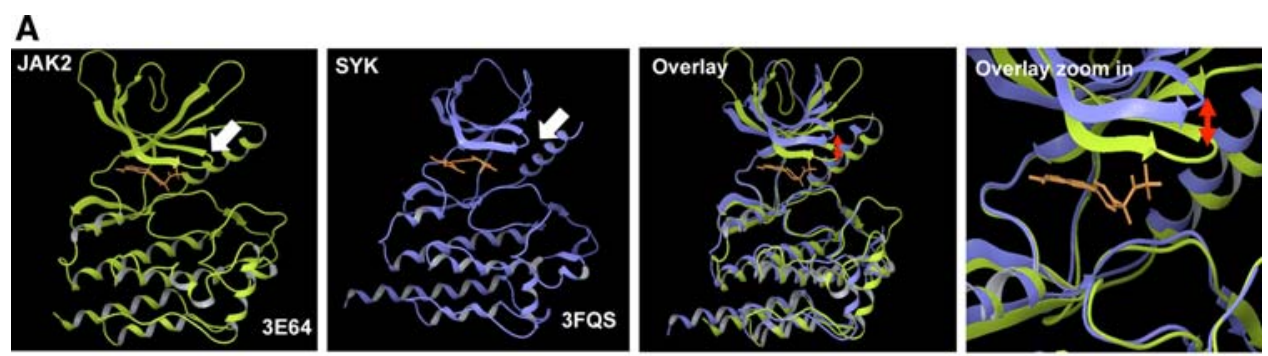

B
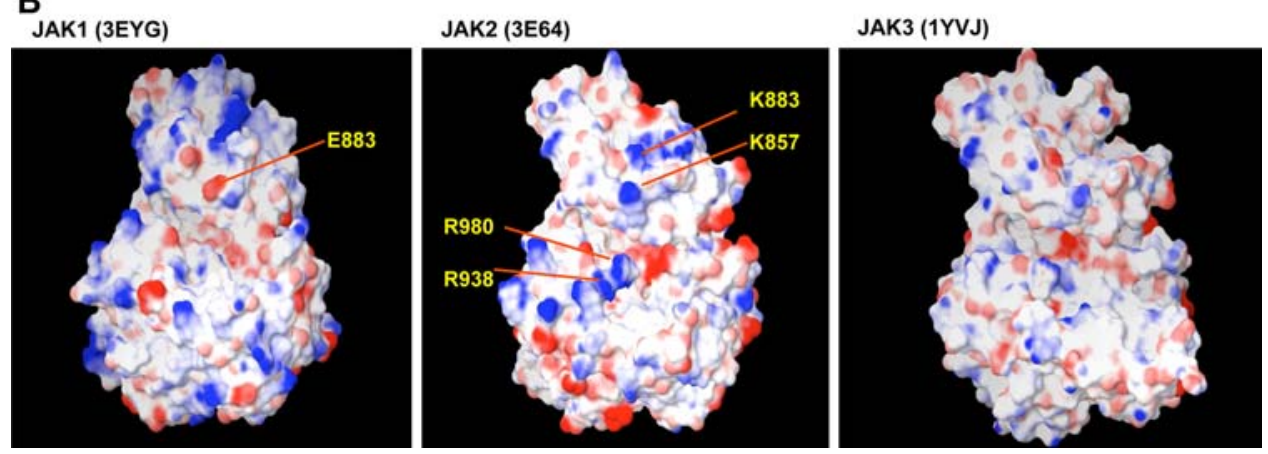

The online version of the original article can be found under doi:10.1007/s12185-010-0531-y.

Y. Hitoshi $(\bowtie) \cdot$ N. Lin · D. G. Payan · V. Markovtsov

Rigel Pharmaceuticals Inc., 1180 Veterans Boulevard,

South San Francisco, CA 94080, USA

e-mail: yhitoshi@rigel.com 